\title{
Morphological Substantiation for the Effectiveness of the Proposed Method of Gastrostomy using a Polypropylene Endoprosthesis
}

\author{
Sergey N. Shurygin, $\mathrm{PhD}, \mathrm{ScD}^{1}$; Alexey G. Vaganov ${ }^{2}$; Archil S. Tsulaya ${ }^{1}$; \\ Sarkis A. Asratyan, $\mathrm{PhD}^{1}$; Ilya V. Sazhin, $\mathrm{PhD}^{1}$; Aleksandr N. Alimov, PhD, $\mathrm{ScD}^{3}$; \\ Leonid V. Safonov, $\mathrm{PhD}^{4 *}$; Dmitriy A. Volkov ${ }^{1}$ \\ ${ }^{I}$ State Budgetary Institution of Healthcare "Clinical Hospital V.M. Buyanova» \\ ${ }^{2}$ State Budgetary Institution of Healthcare of the City of Moscow «City Clinical Hospital No. 29» \\ ${ }^{3}$ Pirogov Russian National Research Medical University \\ ${ }^{4}$ Federal Scientific Center for Physical Culture \& Sports \\ Moscow, the Russian Federation
}

\begin{abstract}
The article discusses the morphological changes in the area of gastrostomy performed by the proposed original method of gastrostomy using an implant in laboratory animals (rabbits). Morphological changes in the tissues at gastrostomy sites were studied on Days 10 and 20 after the proposed method of gastrostomy, in comparison with the classical Witzel gastrostomy and Depage-Janeway gastrostomy using the GIA stapler. Negative morphological changes in the tissues around gastrostomy sites were revealed in the form of necrosis of the microenvironment and disturbance in microcirculation according to the stagnant type, caused by the damaging effect of the surgical suture material at the microscopic level, the least pronounced when using a polypropylene implant. (International Journal of Biomedicine. 2020;10(1):45-49.)
\end{abstract}

Key Words: gastrostomy $\bullet$ original method $\bullet$ polypropylene mesh $\bullet$ histology

\section{Introduction}

Currently, there are about 100 different modifications of gastrostomy. However, the expansion of indications for gastrostomy increases the frequency of its use, which leads to the need for an improvement in technique.(1) Thus, the development of new gastrostomy methods is still relevant, on the one hand contributing to improving the quality of life of patients, and on the other, reducing the risk of complications. Despite the fact that the complication rate for surgical gastrostomy is from $2 \%$ to $8 \%$, and the complication rate for endoscopic gastrostomy is only $1 \%$ to $3 \%$, surgical gastrostomy continues to be used, since the absolute contraindications for performing endoscopic gastrostomy are decompensated stenosis of the stomach, complete obstruction of the pharynx

*Corresponding author: Leonid V. Safonov, PhD. Federal Scientific Center for Physical Culture \& Sports; Moscow, Russia.. E-mail: lsaf@mail.ru and esophagus, obesity grade 3, severe coagulopathy, and other morbidities. ${ }^{(2)}$ At the same time, the expansion of indications for gastrostomy requires the development of new methods for its implementation and assessment of the effectiveness of interventions.

The purpose of the work was to characterize the morphological changes in the tissues around gastrostomy sites in the original method of operation using a polypropylene mesh, in comparison with the classical Witzel gastrostomy and Depage-Janeway gastrostomy using the GIA stapler. The experiment was performed on rabbits.

\section{Materials and Methods}

For the experiment, we selected 18 sexually mature male rabbits ( 3 months old) of the Chinchilla breed, weighing $2500-3400 \mathrm{~g}$. The animals were divided by the method of pair analogues into 3 groups (6 animals each). ${ }^{(3)}$ Group 1 included rabbits that underwent the Witzel's gastrostomy; 
Group 2 included rabbits that underwent the Depage-Janeway gastrostomy using the GIA apparatus; Group 3 included rabbits that underwent the original method of gastrostomy using a polypropylene endoprosthesis (Patent RU No. 2691924 dated 06/18/2019 Bulletin No. 17). ${ }^{(4)}$

For the original method of gastrostomy, we used the synthetic polymer Esfil - Standard of the Lintex company. Esfil is a classic mesh endoprosthesis made from monofilament polypropylene for soft tissue repair, mainly used in herniology. This endoprosthesis combines high rates of biological inertness, resistance to infection and mechanical strength. One of the main disadvantages of the polypropylene implant, which excludes its use in the intraperitoneal plastic of hernias of the anterior abdominal wall, is the development of a massive adhesive process, due to the expressed adhesive properties of the implant, with the possible formation of intestinal fistulas. ${ }^{(3)}$ In our model, this "negative" property of polypropylene material becomes a key advantage, providing a tight attachment of the stomach with a gastrostomy to the anterior abdominal wall (AAW).

The rabbit was chosen for the experiment because it is a standard laboratory animal in the development of new surgical methods. ${ }^{(5)}$ In addition, the rabbit is phylogenetically closer to primates than rodents ${ }^{(6)}$ and is a large enough laboratory animal to monitor physiological changes without euthanasia. The animals were kept in a vivarium in isolated cages with a 12-hour light cycle at a temperature of $18-21^{\circ} \mathrm{C}$.

The method of gastrostomy using a polypropylene mesh was implemented as follows: A median laparotomy was performed; the stomach wall was pulled up to AAW and was taken on two Babcock clamps. A GIA type stapler was placed perpendicular to the greater curvature of the stomach, and a gastric tube (GT) was formed from the stomach wall. From a polypropylene mesh, 2 polypropylene mesh implants were modeled. The first was formed as an oval plate with a central hole. The second implant was formed as a single-layer mesh clutch covering GT. GT was passed through the hole of the first implant, then a second implant was put on it in the form of a clutch. The first implant was fixed to the gastric wall and the second implant to GT, then the two implants were sutured together. Through a hole of $1.5-2 \mathrm{~cm}$ in size, GT was pulled out onto AAW to the left of the midline incision in the projection of the left rectus abdominis muscle.

GT was fixed with sutures to the parietal peritoneum and the muscular aponeurotic layer. The GT end was dissected, 3 fixing sutures were placed, stitching the AAW through, as well as the wall of the stomach together with the first implant attached to it in three places with polypropylene $1 / 0$ thread, ensuring gastropexy. The threads were tied with a knot on the skin, hemostasis was carried out, and then layer-by-layer the laparotomy wound was sutured. Further, in the postoperative period, after 3 weeks, as the implants germinated with connective tissue, which provided an increasingly tight attachment of the stomach wall to AAW, the gastric-fixing sutures were gradually removed.

The animals were withdrawn from the study on Days 10 and 20 after surgery. Tissue fragments were taken from the gastrostomy zone and fixed for at least 2 hours in a $10 \%$ solution of neutral formalin. Further sample processing was carried out by intermediate Blick mixtures. Subsequently, paraffin sections 5-7 $\mu \mathrm{m}$ thick were made, which were stained with H\&E. To identify acidic glycosaminoglycans, which are of particular importance in formation of connective tissue, staining with Alcian blue was used.

Morphometry was performed using the ImageJ-1.45s. We performed quantitative and qualitative analysis of the composition of cell infiltrates in the newly formed connective tissue of the gastrostoma. The severity of the inflammatory reaction was assessed by determining the area of infiltrate in $\mathrm{mm}^{2}$. To determine the prevalence of inflammatory or reparative tendencies, the percentage of cell composition per 100 cells was calculated. Quantitative assessment of reparative changes was determined by counting the resident cells (fibrocytes, fibroblasts, and macrophages), and the inflammatory process was determined by counting the number of the non-resident cells (lymphocytes, neutrophils, and monocytes). The ratio of these cell groups was subsequently presented as a percentage. The relative area of the dermal vessels was calculated as a percentage of the area of the dermal connective tissue in one field of view.

The work with animals was carried out in accordance with the principles of humanism laid down in the directives of the European Community (86/609/EEC) and the Declaration of Helsinki, in accordance with the "Animal experimentation legislations".

Statistical analysis was performed using the statistical software «Statistica». (v10.0, StatSoft, USA) and Microsoft Excel 2007. Baseline characteristics were summarized as frequencies and percentages for categorical variables and as mean \pm SEM for continuous variables. The Mann-Whitney (U Test) was used to compare the differences between the two independent groups. A probability value of $P<0.05$ was considered statistically significant.

\section{Results}

\section{Day 10 after surgery}

In Group 1, we found healing of the tissue defect with the formation and maturation of granulation tissue, and its reorganization, closing of the defect with the epidermis and scar formation in the zone of the imposed gastrostoma from the side of AAW. In the epidermis, all layers characteristic of intact skin were differentiated. An increase in the thickness of the epidermis was caused by a thickening of the prickly and granular layers, while the epithelial papillae were virtually absent, due to which the relief of the epithelium was smoothed. Skin appendages were not numerous (Fig.1.1). In the deep layers of the newly formed connective tissue, we found randomly located, newly formed, thickened collagen fibers surrounded by fibroblasts. On a number of micropreparations, a lymphocytic infiltrate separating the fibers was visualized. In the area adjacent to the suture material, cellular detritus was formed as a result of tissue necrosis (Fig.1.2).

In Group 2, we found the formation of a thicker epidermal layer, due to an increase in the thickness of the granular and horny layers. The appendages of the skin were more numerous and represented not only by hair follicles, 
but also by the terminal sections of the sebaceous glands. Subepidermal vessels were significantly dilated. The red blood cells in them were in a state of sludge. Venous lumen was characterized by the dissociation of blood plasma, and signs of perivascular edema were noted (Fig.1.3). The same signs of impaired microcirculation by stagnant type were noted in the deep sections of the connective tissue of the gastrostoma. The dilated venules, with dissociated plasma, were surrounded with numerous clusters of non-resident cells. In the area adjacent to the suture material, less pronounced cellular detritus, due to tissue necrosis, was formed.

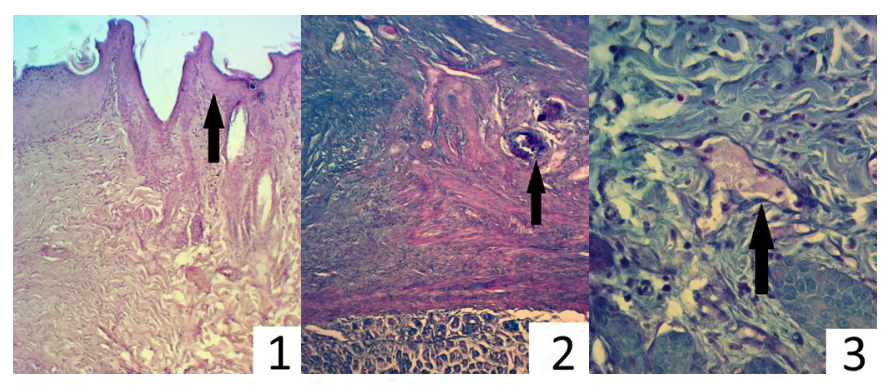

Fig.1. The area of the anastomosis at the transition zone between the stratified squamous keratinized epithelium of $A A W$ and simple columnar epithelium of the stomach; Day 10 after surgery.

1.1.The Witzel's gastrostomy. Skin epidermis with few hair follicles. 1.2.The Witzel's gastrostomy. Cellular detritus in the newly formed connective tissue, located in the deep sections of the anastomosis, closer to the gastric mucosa.

1.3.The Depage-Janeway gastrostomy. The dissociation of blood plasma in the expanded venule of newly formed connective tissue with a large number of non-resident cells.

In Group 3, a complicated running of the epidermal basement membrane was noted. It acquired a convoluted character with the formation of numerous papillary papillae of complex shape that layered on top of each other, due to which the entire surface of the epithelium was corrugated. As in Group 2, the granular and horny layers were more pronounced, in comparison with Group 1, and skin appendages were more numerous (Fig.2.1).The subepidermal connective tissue was filled with dense inflammatory-cellular infiltrates; however, unlike in Group 2 , we did not reveal paretically dilated subepidermal vessels. In the deep sections of the anastomosis, around artifacts associated with the threads of the polypropylene mesh, already at this time, the ordered dense intercellular connective tissue structures were determined. In the area adjacent to the suture material, we did not observe accumulations of cellular detritus characteristic of Groups 1 and 2.

\section{Day 20 after surgery}

In Group 1, in the zone of superimposed gastrostoma from the side of AAW, we found the completion of reepithelialization of the contact site of the stomach epithelium and skin epidermis, with complete differentiation of the epidermal layers. The uneven thickness of the epidermis persisted. A local increase in the thickness of the epidermis was caused by a thickening of the prickly and granular layers. Epithelial papillae were more numerous, and in their form were close to those of intact rabbits. An increase in the number of skin appendages, represented by numerous hair follicles collected in groups, was noted. Collagen fibers filled the entire volume of the zone of the formed scar and had a convoluted course. The underlying connective tissue scaffold was more mature, compared to the morphological picture of the previous period in this group. In the deep layers of the newly formed connective tissue, chaotically located collagen fibers were preserved, alternating with the connective tissue sites depleted in acidic glycosaminoglycans. Cellular detritus, previously formed at the site of surgical sutures, was surrounded by a connective tissue capsule with sites of lymphocytic infiltrate. Foci were preserved in this group and Group 2, and fresh cell detritus was contained.

In Group 2, as in Group 1, the thicker epidermal layer, as it approaches the zone of artificial transition into a simple columnar epithelium of the stomach, was smoothed and lost the horny layer, as well as the appendages of the skin. Numerous subepidermal vessels, characteristic for Day 10, became empty after Day 20. Similar changes on the part of the microvasculature were noted in the deep sections of the connective tissue of the gastrostoma (Fig.2.2).

In Group 3, a complicated running of the epidermal basement membrane persisted over the entire surface of the epidermis adjacent to the anastomosis zone. Moreover, it spread to the transition zone of direct contact with the epithelium of the stomach (Fig.2.3). In this place, the epithelium was reduced to a single or double layer, in some places acquiring a uni-layer, multirow, prismatic character. However, keratinization processes persisted. In the deep sections of the anastomosis, numerous ordered, dense, intercellular connective tissue structures were observed. Between the fibers and in the immediate vicinity of artifacts associated with the elements of the polypropylene network, clusters of cells of inflammatory-cell infiltrate, mainly represented by resident cells, were determined, which characterized the high activity of reparative processes. No sites of necrosis or accumulations of cellular detritus were detected.

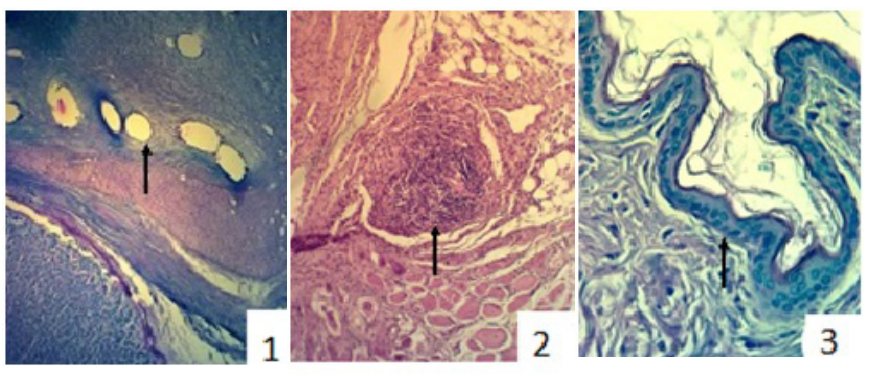

Fig.2. The area of the anastomosis at the transition zone between the stratified squamous keratinized epithelium of $A A W$ and simple columnar epithelium of the stomach.

2.1. Gastrostomy using a polypropylene mesh. Artifacts in the threads of the polypropylene mesh. There are no sites of necrosis.

2.2. The Depage-Janeway gastrostomy. The encapsulated area of necrosis in the deep layers of the anastomosis adjoining to abdominal striated muscles.

2.3. Gastrostomy using a polypropylene mesh. Epithelial metaplasia in the transition region between the skin epidermis and simple columnar epithelium of the stomach.

According to a morphometric study, 10 days after surgery the largest number of inflammatory infiltrate cells in 
the field of view was characteristic of Group 1 and Group 2 micropreparations, due to the accumulation of non-resident cells (Table 1). The identified features characterized the high activity of the acute phase of inflammation. Later, 20 days after the operation, the situation was changed: the maximum cell area was observed in animals of Group 3, where the original method of gastrostomy with a polypropylene mesh was used. The increase in cell area was associated with a high percentage of resident cells, which amounted to $23.8 \%$, while in Groups 1 and 2 these indicators were $15.7 \%$ and $13.4 \%$, respectively.

\section{Table 1.}

Morphometric indicators of connective tissue in the anastomotic zone

\begin{tabular}{|l|c|c|c|c|}
\hline \multicolumn{1}{|c|}{ Variable } & Day & Group 1 & Group 2 & Group 3 \\
\hline $\begin{array}{l}\text { The number of } \\
\text { inflammatory } \\
\text { infiltrate cells }\end{array}$ & 10 & $509 \pm 34$ & $456 \pm 35$ & $401 \pm 35^{* »}$ \\
\cline { 2 - 5 } & 20 & $248 \pm 15$ & $282 \pm 25$ & $356 \pm 30^{* \wedge}$, \\
\hline \multirow{2}{*}{ Resident cells.\% } & 10 & $64.5 \pm 4.1$ & $62.8 \pm 4.1$ & $50.1 \pm 5.2^{* \wedge}$ \\
\cline { 2 - 5 } & 20 & $84.3 \pm 6.1$ & $86.6 \pm 6.9$ & $76.2 \pm 5.9^{* \wedge} "$ \\
\hline \multirow{2}{*}{ Non-resident cells.\% } & 10 & $35.5 \pm 4.1$ & $37.2 \pm 4.1$ & $49.9 \pm 5.2^{* \wedge}$ \\
\cline { 2 - 5 } & 20 & $15.7 \pm 6.1$ & $13.4 \pm 6.9$ & $23.8 \pm 5.9^{* \wedge} "$ \\
\hline \multirow{2}{*}{$\begin{array}{l}\text { Area of inflammatory } \\
\text { cell infiltrate }\end{array}$} & 10 & $0.68 \pm 0.07$ & $0.63 \pm 0.05$ & $1.08 \pm 0.09^{* \wedge}$ \\
\cline { 2 - 5 } & 20 & $0.32 \pm 0.02$ & $0.37 \pm 0.03$ & $0.89 \pm 0.07^{* \wedge} "$ \\
\hline $\begin{array}{l}\text { The relative area of } \\
\text { the blood vessels of } \\
\text { the dermis }\end{array}$ & 10 & $10.15 \pm 0.08$ & $12.43 \pm 1.1$ & $8.72 \pm 0.71^{* \wedge}$ \\
\cline { 2 - 5 } & 20 & $6.98 \pm 0.06$ & $9.5 \pm 0.78$ & $5.44 \pm 0.36^{* \wedge »}$ \\
\hline
\end{tabular}

* - P<0.05 compared to Group $1 ; \wedge^{\wedge}-P<0.05$ compared to Group 2; “ - $P<0.05$ between Days 10 and 20 in Group 3

On Day 20 after surgery, the largest specific area of the microvasculature of the newly formed connective tissue was noted in Group 2; it was 75\% higher than in Group 3 and 36\% higher than in Group 1. This indicator was inversely proportional to the number of resident cells in the cell infiltrates of the newly formed connective tissue.

\section{Discussion}

This study confirmed the development of negative necrotic changes around the suture during the formation of anastomoses. In the early stages, these changes are accompanied by the formation of cellular detritus, and in the later stages, the appearance of thick connective tissue capsules with gross fibro-degenerative changes and signs of inflammatory reactions associated with the "sawing effect" of the thread during tension. ${ }^{(7)}$

In addition, the alterative processes are caused not only by mechanical damage to tissues or the penetration of infectious agents, but also by a violation of microcirculation, which is of great importance for the formation of a full anastomosis between the hollow organs. ${ }^{(8)}$ As known, there are significant differences in the nature of the microvasculature of the skin and the stomach wall, which provokes a violation of hemodynamics in the gastrostomy zone, which, against the background of inflammatory reactions, is accompanied by a violation of tissue metabolism and an increase in lipid peroxidation markers. ${ }^{(9)}$ Activation of apoptosis mechanisms in endotheliocytes under these conditions may inhibit the expansion of newly formed vessels and inhibit neoangiogenesis. Thus, the circulatory disturbance observed in our study in the group of laboratory animals with the Depage-Janeway gastrostomy can play a significant role in the development of a complex of wound complications that can be minimized by performing the original method of gastrostomy with a polypropylene implant.

The revealed microcirculatory disorders and necrosis in the area of suture material (in the Witzel and Depage-Janeway models of gastrostomy) are leveled using a polypropylene mesh. In the literature, there are indications of the features of reparative processes under the influence of polypropylene implants, namely the activation of fibroblasts on the fifth day after the operation, the formation of granulation tissue with many thin-walled vessels, and collagenization of the interstitial matrix on the 10th day. By the 14th day, according to published data, the interstitial inflammatory infiltration by lymphocytes and macrophages is significantly reduced, ${ }^{(10)}$ which is consistent with the data obtained in our study.

Thus, the following conclusions can be drawn:

1. The morphological changes in the tissue ensemble in the gastrostoma zone, which determine the development of complications in the postoperative period, are usually based on the surgical technique for performing a gastrostomy.

2. Marked negative morphological changes in the gastrostomazone in the form of necrosis of the microenvironment and disturbances of microcirculation according to the stagnant type are caused by the damaging effect of the surgical suture material and occur in groups of animals that underwent gastrostomy without the use of a polypropylene implant.

3. The use of a polypropylene implant significantly reduces the severity of necrotic changes in tissues in the area of suture material, primarily due to tissue remodeling caused by the specificity of fibrillogenesis under the influence of polypropylene, which contributes to more reliable attachment of the gastrostoma and lower mechanical load on the ligatures due to an increase in the strength of the newly formed connective tissue.

\section{Competing Interests}

The authors declare that they have no competing interests.

\section{References}

1. Tanaka T, Ueda T, Yokoyama T, Sadamitsu T, Yoshimura A, Horiuchi H, et al. Laparoscopic Percutaneous Endoscopic Gastrostomy Is Useful for Elderly. JSLS. 2019 Apr-Jun;23(2). pii: e2019.00011. doi: 10.4293/JSLS.2019.00011.

2. Khoroshilov I.E. [Endoscopic gastrostomy: 35 years of use in the clinic]. Gastroenterologia Sankt-Peterburga. 2015; (3-4) M17-M17a.[Article in Russian].

3. Peeters E, Spiessens C, Oyen R, De Wever L, Vanderschueren D, Penninckx F, Miserez M. Sperm motility after laparoscopic inguinal hernia repair with lightweight 
meshes: 3-year follow-up of a randomised clinical trial. Hernia. 2014 Jun;18(3):361-7. doi: 10.1007/s10029-0121028-9.

4. Vaganov AG, Tsulaya AS, Shurygin SN. Patent RU No. 2691924 dated 06/18/2019 Bulletin No. 17. [In Russian].

5. Rybakova AV, Makarova MN, Makarov VG. [Using rabbits in pre-clinical trials. Mezhdunarodnyi vestnik veterinarii. 2016;(4):113-117. [Article in Russian].

6. Mishina ES, Zatolokina MA, Netyaga AA, Klimova LG, Zhukovskiy VA. [Reactive changes of connective tissue anterior abdominal wall in the early postoperative period with using experimental samples net endoprosthesis antibacterial coating]. Modern problems of science and education. 2015;(2 Part 1):55. [Article in Russian].

7. Bontsevich DN, Golubev OA. [Experimental use of improved suture material]. Problemy zdorov'ya i ekologii. 2004;(2):141-144. [Article in Russian].

8. Wang ZG1, Huang YD, Cheng KL, Cai XB, Wu Z, Zhan JD. [Influence of blood supply of the esophageal and gastric stumps on anastomotic healing after esophagogastrostomy in rabbits].Di Yi Jun Yi Da Xue Xue Bao. 2004 Mar;24(3):345-6, 351. [Article in Chinese].

9. Markos'yan SA, Vlasov AP. [Experimental evaluation of tissues' changes of double layer small bowel anastomosis at various age]. University proceedings. Volga region. Medical sciences. 2018;(1):18-26. [Article in Russian].

10. Qu S, Xia J, Yan J, Wu H, Wang H, Yi Y, et al. In vivo and in vitro assessment of the biocompatibility and degradation of high-purity $\mathrm{Mg}$ anastomotic staples. J Biomater Appl. 2017 Mar;31(8):1203-1214. doi: 10.1177/0885328217692948. 